\title{
Coupled channel effect in elastic scattering and fusion for ${ }^{6,7} \mathbf{L i}+{ }^{28} \mathbf{S i}$
}

\author{
Mandira Sinha ${ }^{1}$, a , Subinit Roy ${ }^{1}$, P. Basu ${ }^{1}$, H. Majumdar ${ }^{2}$, S. Santra ${ }^{3}$, V.V. Parkar ${ }^{4}$, K.S. Golda ${ }^{5}$, and S. Kailas ${ }^{3}$ \\ 1 Nuclear Physics Division, Saha Institute of Nuclear Physics, Kolkata - 700064, INDIA \\ 2 R.K.Mission Vivekananda University, Belur Math, Howrah-711202, INDIA \\ 3 Nuclear Physics Division, Bhabha Atomic Research Centre, Mumbai- 400085, INDIA \\ 4 Departamento de Fìsica Aplicada, Universidad de Huelva, E-21071 Huelva, SPAIN \\ 5 Inter-University Accelerator Centre, New Delhi- 110067, INDIA
}

\begin{abstract}
The fusion excitation and elastic angular distribution were measured for ${ }^{6,7} \mathrm{Li}+{ }^{28} \mathrm{Si}$ from below to above Coulomb barrier $\left(\leq 3 \mathrm{~V}_{b}\right)$ energies. The barrier distribution derived from the fusion data was found to be broad and asymmetric at the sub-barrier region, compared to 1D BPM estimation. Effect of rotational coupling on fusion was found to be not so dominant. Phenomenological optical potential parameters, with surface and volume type imaginary potentials, were obtained from $\mathrm{ftting}$ of elastic scattering data and energy dependence of real and imaginary surface strengths were investigated around the barrier. CDCC calculations considering only breakup of projectile were performed for ${ }^{6,7} \mathrm{Li}+{ }^{28} \mathrm{Si}$ with the elastic scattering data, using the code FRESCO. The effects of breakup of projectile on elastic cross section do not agree with the energy dependence of real and imaginary strength with volume type imaginary potential around the barrier.
\end{abstract}

\section{Introduction}

The reaction dynamics of loosely bound projectiles with light mass target at near barrier energies is very important and not yet fully understood. The measured fusion cross section at above barrier energies showed suppression [1, 2] when compared with one dimensional barrier penetration model (1D BPM), whereas the same were enhanced at sub barrier energies [2,3]. On the otherhand, the near barrier behaviour of the interaction potential for ${ }^{6,7} \mathrm{Li}+{ }^{28} \mathrm{Si}$ $[4,5]$ as a function of energy was found to be quite different compared to the observations with heavier targets. Recently, a simultaneous description of the elastic, fusion and reaction cross sections has been made by A. Gomez Camacho etal. for the same system [6] with phenomenological approach, where the nuclear polarization potential is split into a volume and a surface part to understand the energy dependence of the OM potential in terms of different polarization potentials. So for such light systems with reduced Coulomb strength, the inf uence of breakup and other direct reactions (e.g. transfer) on fusion, elastic scattering and barrier distribution is def nitely interesting to probe the interplay of the different processes. With this motivation we present the results of the measurements and analysis of elastic cross sections for the ${ }^{6,7} \mathrm{Li}+{ }^{28} \mathrm{Si}$ systems. The effect of breakup on elastic was also observed in the energy range with $\mathrm{E} / V_{b}=0.9$ to 3.0 , employing CDCC calculation.

\footnotetext{
a e-mail: mandira.sinha@saha.ac.in
}

\section{Experimental details and Analysis}

In this perspective we have measured elastic angular distributions for ${ }^{6,7} \mathrm{Li}+{ }^{28} \mathrm{Si}$ at certain energies in the interval $E_{l a b}=11.5-26 \mathrm{MeV}$. The above barrier elastic scattering measurements were performed alongwith the measurement of above barrier fusion cross section for ${ }^{7} \mathrm{Li}+{ }^{28} \mathrm{Si}$ by evaporation $\alpha$ - method [1]. Experiment was performed at the 15 UD Pelletron facility of Inter University Accelerator Centre (IUAC), New Delhi with ${ }^{7} \mathrm{Li}$ beam at energies $E_{l a b}=$ 16,21 and $26 \mathrm{MeV}$ and ${ }^{6} \mathrm{Li}$ beam at energies $E_{l a b}=21$ and $26 \mathrm{MeV}$. The Si target of thickness $150 \mu \mathrm{g} / \mathrm{cm}^{2}$ sandwiched between two Au layers was used. The elastic angular distribution was measured from the $2 \mathrm{D}$ spectra (E Vs $\Delta \mathrm{E}$ ) of two telescopes ( $\triangle \mathrm{E}: 25 \mu \mathrm{m}, \mathrm{E}: 300 \mu \mathrm{m})$ placed at different angles, varying from $\theta_{l a b}=15.5^{\circ}$ to $94.5^{\circ}$, in small steps. Two monitor detectors $(300 \mu \mathrm{m})$ were mounted at forward angles $\pm 9.8^{\circ}$ (f xed to the wall of the chamber) with respect to beam axis and were used to monitor the beam axis and also for absolute normalisation purposes. The solid angles subtended by the telescopes and monitor detectors at the target centre were respectively $1.18 \times 10^{-4} \mathrm{sr}, 1.36 \times 10^{-4}$ $s r$ and $6.5 \times 10^{-6} s r$ respectively. Angular resolution $(\Delta \theta)$ of each telescope detector was about $\sim 0.7^{\circ}$.

A separate experiment was carried out using the General Purpose Scattering Chamber (GPSC) at the 14 UD BARC-TIFR Pelletron facility in Mumbai for the measurements of near barrier elastic scattering for ${ }^{7} \mathrm{Li}+{ }^{28} \mathrm{Si}$ system at $E_{l a b}=11.5$ and $13 \mathrm{MeV}$. A self supporting Si target of thickness $150 \mu \mathrm{g} / \mathrm{cm}^{2}$ was used. During the experiment the beam current $\left({ }^{7} \mathrm{Li}^{2+}\right)$ was varied from $2-20 \mathrm{pnA}$. The elastically scattered particles were detected using the tele- 
scope arrangements as discussed earlier. Two telescopes were used viz., ( $\triangle \mathrm{E}: 25 \mu \mathrm{m}, \mathrm{E}: 300 \mu \mathrm{m})$ and $(\Delta \mathrm{E}: 15 \mu \mathrm{m}, \mathrm{E}$ : $500 \mu \mathrm{m})$. The dectectors were placed on the rotating arm inside the scattering chamber with angular seperation of $10^{\circ}$. Two monitor detectors $(2 \mathrm{~mm}, 3 \mathrm{~mm})$ were mounted at $\pm 15^{\circ}$ w.r.t. the beam axis. The solid angles subtended by the telescopes and monitor detectors at the target centre were respectively $9.9 \times 10^{-4} \mathrm{sr}, 1.7 \times 10^{-4} \mathrm{sr}$ and $4.7 \times 10^{-6}$ $s r$. The angular resolution $(\Delta \theta)$ of all the detectors was better than $\sim 0.4^{\circ}$. We also previously measured total fusion cross sections in the energy range $E_{l a b}=11.5-26 \mathrm{MeV}$ for ${ }^{6,7} \mathrm{Li}+{ }^{28} \mathrm{Si}[1]$ at the BARC-TIFR and IUAC Pelletron facilities. Moreover separate experiments were performed at sub-barrier energies from 7-11.5 MeV for ${ }^{6,7} \mathrm{Li}+{ }^{28} \mathrm{Si}[2,3]$ at IOP Pelletron facility, Bhubaneswar.

The elastic scattering data from 7.5 to $26 \mathrm{MeV}$ were then analysed using the phenomenological Woods-Saxon potential employing the code ECIS94. The scattering data at other lower energies were taken from ref [4]. In the code ECIS94, rotational coupling of $2^{+}$state of the target with $\beta=-0.407$ was taken into account. Two sets of OM potential parameters with surface (OM1) and volume type(OM2)imaginary potentials were obtained from the best $\mathrm{ft}$ procedure. In order to obtain the best $\mathrm{ft} \mathrm{OM}$ potential parameters, all six parameters were varied simultaneously. For surface type imaginary potential (OM1), the volume imaginary potential $W_{v}(R)$ was kept f xed with the energy independent parameter set of $W_{F}=50 \mathrm{MeV}, r_{F}=1.0 \mathrm{fm}$, $a_{F}=0.4 \mathrm{fm}$. The extracted reaction cross sections using both sets of potentials are found to be similar, however values are larger for ${ }^{6} \mathrm{Li}$ than ${ }^{7} \mathrm{Li}$. The energy dependence of effective potential parameters OM2 (Table 1 and Table 2), in a wide range of energies, is explored at the crossing radii as shown in Fig.3, Fig.4. The real and imaginary strength are evaluated at average crossing radius and is found to be almost independent of energy for $E_{l a b}>1.5 V_{b}$, although it starts to drop with decreasing energy towards the barrier. The real potential behaviour for ${ }^{6} \mathrm{Li}$ is almost similar to that of ${ }^{7} \mathrm{Li}$, however rate of decrease seems to be higher for ${ }^{6} \mathrm{Li}$ than ${ }^{7} \mathrm{Li}$. Also to connect the energy dependent behaviours of the real and the imaginary potential strengths at the surface using the dispersion relation was not succesful. Our results differ from the previous observations [7] for the same systems ${ }^{6,7} \mathrm{Li}+{ }^{28} \mathrm{Si}$ where optical potentials were obtained by the double-folding procedure. These authors found almost energy independent normalisation factors $\left(N_{R}\right)$ for real potentials, for both ${ }^{6} \mathrm{Li}$ and ${ }^{7} \mathrm{Li}$, where as normalisation factors $\left(N_{I}\right)$ for imaginary potentials showed marked decreasing tendency towards the barrier for ${ }^{7} \mathrm{Li}$ compared to ${ }^{6} \mathrm{Li}$. Threshold behaviour of potentials (real and imaginary) is somewhat different from those usually found for cases involving medium and heavy mass targets Ref. [9-12].

The effect of breakup on elastic scattering was investigated within the continuum discretized coupled-channels (CDCC) framework using the code FRESCO [8] (Versions FRXP.18 and FRES 2.4). In CDCC calculation two body cluster structures of ${ }^{6} \mathrm{Li}(\alpha+\mathrm{d})$ and ${ }^{7} \mathrm{Li}(\alpha+\mathrm{t})$ were considered. The diagonal and non-diagonal coupling potentials for ${ }^{6} \mathrm{Li}+{ }^{28} \mathrm{Si}$ and ${ }^{7} \mathrm{Li}+{ }^{28} \mathrm{Si}$ were generated from the em-
Table 1. Optical Model (OM2) phenomenological potential parameters for ${ }^{7} \mathbf{L i}+{ }^{28} \mathbf{S i}$ with the extracted reaction cross sections as function of energy.

\begin{tabular}{|c|c|c|c|c|c|c|}
\hline $\begin{array}{c}E_{l a b} \\
(\mathrm{MeV})\end{array}$ & $\begin{array}{c}V_{0} \\
(\mathrm{MeV})\end{array}$ & $\begin{array}{c}r_{0} \\
(\mathrm{fm})\end{array}$ & $\begin{array}{c}a_{0} \\
(\mathrm{fm})\end{array}$ & $\begin{array}{c}W_{v} \\
(\mathrm{MeV})\end{array}$ & $\begin{array}{c}r_{v} \\
(\mathrm{fm})\end{array}$ & $\begin{array}{c}a_{v} \\
(\mathrm{fm})\end{array}$ \\
\hline \hline 8.5 & 5.1 & 1.12 & 0.808 & 5.96 & 1.17 & 0.786 \\
10 & 10.2 & 1.12 & 0.788 & 10.32 & 1.17 & 0.786 \\
11.5 & 16.9 & 1.08 & 0.766 & 5.87 & 1.16 & 0.783 \\
13 & 17.3 & 1.08 & 0.766 & 16.92 & 1.16 & 0.783 \\
16 & 19.7 & 1.08 & 0.766 & 23.29 & 1.16 & 0.783 \\
21 & 20.8 & 1.08 & 0.766 & 15.93 & 1.16 & 0.783 \\
26 & 49.6 & 1.07 & 0.717 & 20.46 & 1.16 & 0.700 \\
\hline
\end{tabular}

Table 2. Optical Model (OM2) phenomenological potential parameters for ${ }^{6} \mathbf{L i}+{ }^{28} \mathbf{S i}$ with the extracted reaction cross sections as function of energy.

\begin{tabular}{|c|c|c|c|c|c|c|}
\hline $\begin{array}{c}E_{l a b} \\
(\mathrm{MeV})\end{array}$ & $\begin{array}{c}V_{0} \\
(\mathrm{MeV})\end{array}$ & $\begin{array}{c}r_{0} \\
(\mathrm{fm})\end{array}$ & $\begin{array}{c}a_{0} \\
(\mathrm{fm})\end{array}$ & $\begin{array}{c}W_{v} \\
(\mathrm{MeV})\end{array}$ & $\begin{array}{c}r_{v} \\
(\mathrm{fm})\end{array}$ & $\begin{array}{c}a_{v} \\
(\mathrm{fm})\end{array}$ \\
\hline \hline 7.5 & 5.7 & 1.02 & 0.822 & 3.79 & 1.19 & 0.865 \\
9 & 19.6 & 1.01 & 0.812 & 9.16 & 1.19 & 0.845 \\
11 & 20.1 & 1.01 & 0.809 & 15.41 & 1.17 & 0.776 \\
13 & 33.9 & 1.01 & 0.792 & 22.39 & 1.17 & 0.776 \\
16 & 45.9 & 1.01 & 0.793 & 38.24 & 1.17 & 0.733 \\
21 & 57.3 & 1.01 & 0.766 & 42.98 & 1.16 & 0.675 \\
\hline
\end{tabular}

perical $\alpha+{ }^{28} \mathrm{Si}$ [13], $\mathrm{d}+{ }^{28} \mathrm{Si}$ [14] and $\mathrm{t}+{ }^{28} \mathrm{Si}$ [15] optical potentials using the single folding approach. No target excitation was included in the CDCC calculations. In our calculation we discretized the continua following the scheme presented in Ref. [16]. However, for ${ }^{6} \mathrm{Li}+{ }^{28} \mathrm{Si}, \mathrm{L}=0,1,2$ and 3 nonresonant and resonant states were included in the calculation although the resonance state at $5.65 \mathrm{MeV}\left(1^{+}\right)$ excitation energy in $\mathrm{L}=2$ continuum was excluded. Excitation of ${ }^{6} \mathrm{Li}$ upto $12 \mathrm{MeV}$ was considered at higher bombarding energies. At lower bombarding energies the upper limit of excitation energy was suitably truncated. To match the elastic angular distribution at the highest bombarding energy for ${ }^{6} \mathrm{Li}+{ }^{28} \mathrm{Si}$ the input real and imaginary potentials for $\alpha+{ }^{28} \mathrm{Si}$ were normalized with factors of $N_{R}=0.7$ and $N_{I}=2.8$. The normalization factors were kept $\mathrm{f}$ xed for lower bombarding energies. The resultant predictions are shown in Fig. 1. Similar normalization values had earlier been used for ${ }^{6,7} \mathrm{Li}+{ }^{59} \mathrm{Co}$ by Beck, et al. [17]. For ${ }^{7} \mathrm{Li}+{ }^{28} \mathrm{Si}$, $\alpha$ and triton breakup from the bound excited state $(0.47$ $\mathrm{MeV})$ as well as the resonant and non-resonant states of realtive angular momentum of $\mathrm{L}=0,1,3$ in the continuum were considered in the coupling scheme. The coupling to the continuum for $\mathrm{L}=2$ had been neglected because of its insignif cant contribution as observed by [18]. The continuum up to the excitation energy of $9.32 \mathrm{MeV}(\mathrm{k}=0.75$ $\mathrm{fm}^{-1}$ ) was considered. For ${ }^{7} \mathrm{Li}+{ }^{28} \mathrm{Si}$, the normalisation factors for the real and imaginary input potentials for $\alpha+{ }^{28} \mathrm{Si}$ 


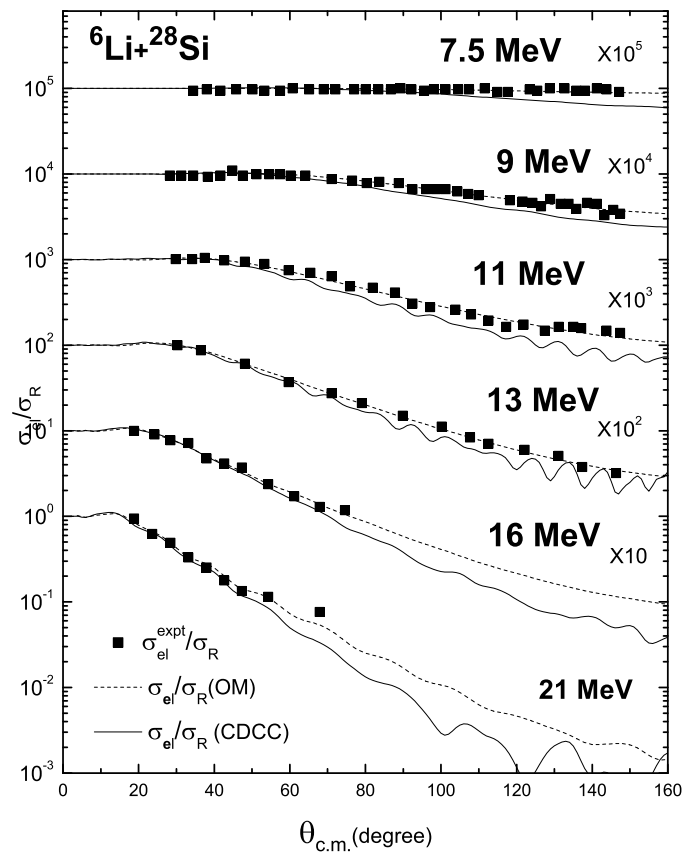

Fig. 1. Elastic angular distribution calculated with OM2 potential (dashed line) and CDCC calculation (solid line) for ${ }^{6} \mathrm{Li}+{ }^{28} \mathrm{Si}$ compared with measured data.

as well as for $\mathrm{t}+{ }^{28} \mathrm{Si}$ were set at $N_{R}=0.7, N_{I}=2.8$ by reproducing the elastic angular distribution at the highest bombarding energy of $26 \mathrm{MeV}$. The same potential normalizations were used for all other incident energies. It is to be noted that a normalization factor of $N_{R}=0.6$ had been used for $\alpha+{ }^{28} \mathrm{Si}$ and $\mathrm{t}+{ }^{28} \mathrm{Si}$ real emperical potentials in ${ }^{7} \mathrm{Li}+{ }^{28} \mathrm{Si}$ by Zerva, et al.[5]. The CDCC predictions from the present work are shown in Fig. 2.

In order to observe the energy dependences of real and imaginary components of the effective potential, i.e. the 'bare' potential plus the polarization potential due to breakup, were extracted from the elastic angular distributions predicted by CDCC using a search routine. The energy dependence of resulting potential values evaluated at average crossing radius $\left(R_{a v}\right)$ are plotted and compared with those obtained by ftting the experimental elastic angular distributions in Figs. 3 and 4.

\section{Results}

The comparison of measured elastic angular distributions with those predicted by CDCC model in Figs. 1 and 2 show that at higher incident energies, coupling to breakup channel describes the data well. But at lower energies near the Coulomb barrier, the model predictions clearly underestimate the data. The model calculations appear to describe

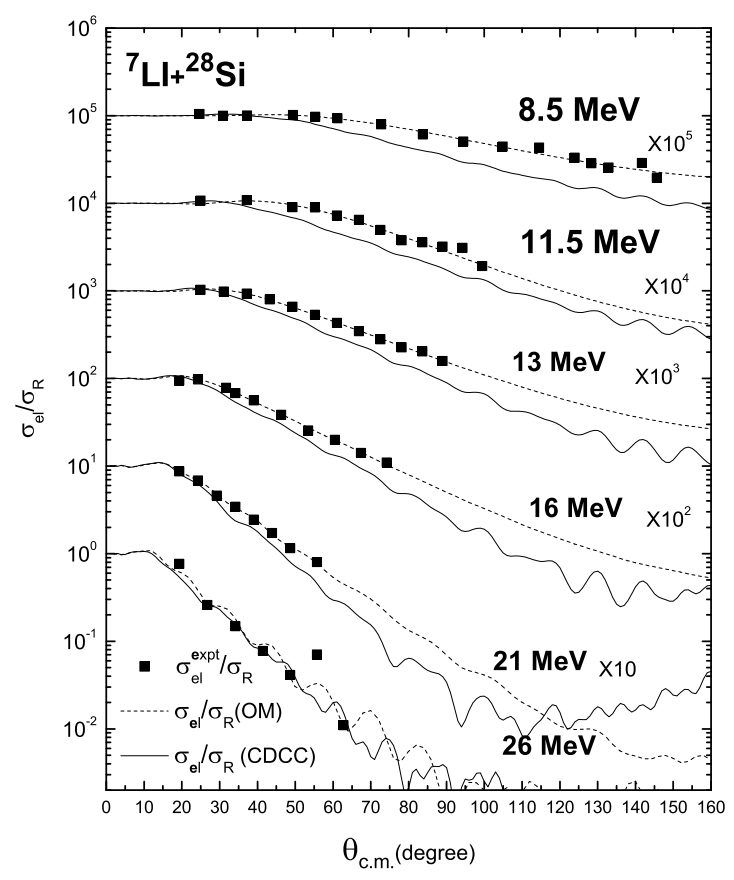

Fig. 2. Elastic angular distribution calculated with $\mathrm{OM} 2$ potential (dashed line) and CDCC calculation (solid line) for ${ }^{7} \mathrm{Li}+{ }^{28} \mathrm{Si}$ compared with measured data.
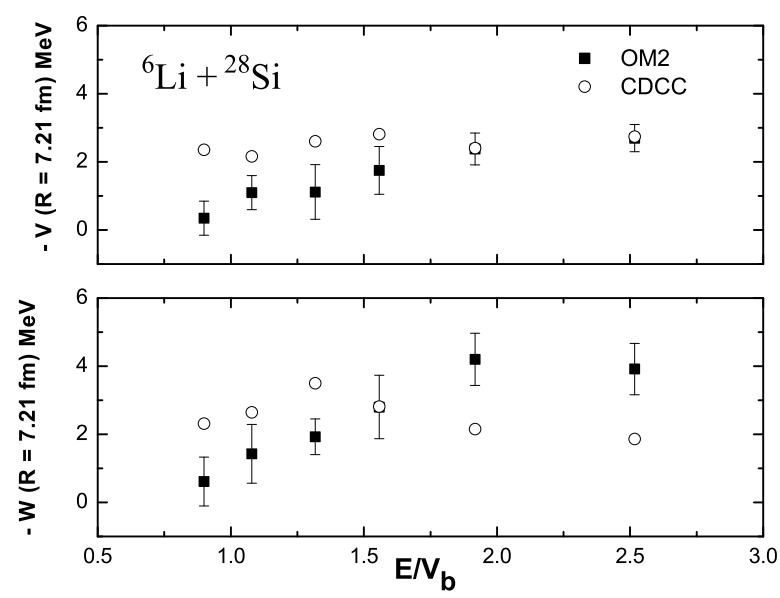

Fig. 3. Threshold behaviour of real and imaginary strength at average crossing radius for $\mathrm{OM} 2$ potential (solid square) and $\mathrm{CDCC}$ calculation (open circle) for ${ }^{6} \mathrm{Li}+{ }^{28} \mathrm{Si}$ 

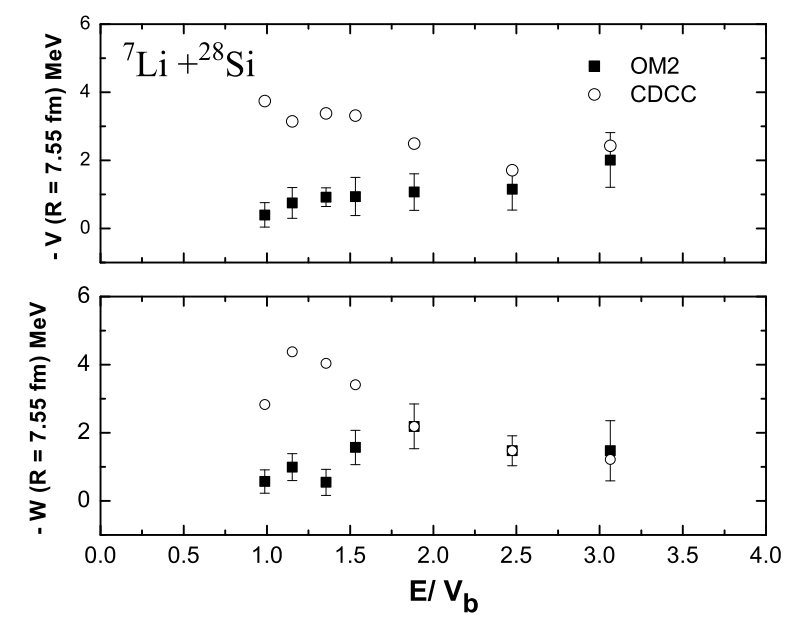

Fig. 4. Threshold behaviour of real and imaginary strength at average crossing radius for $\mathrm{OM} 2$ potential (solid square) and $\mathrm{CDCC}$ calculation (open circle) for ${ }^{7} \mathrm{Li}+{ }^{28} \mathrm{Si}$

the angular distributions of ${ }^{6} \mathrm{Li}+{ }^{28} \mathrm{Si}$ better than those of ${ }^{7} \mathrm{Li}+{ }^{28} \mathrm{Si}$ system. The underpredictions in case ${ }^{7} \mathrm{Li}+{ }^{28} \mathrm{Si}$ are quite signif cant. It is observed from Figs. 3 and 4 that the volume strengths of the optical model potential for both the systems show very similar trend with decreasing bombarding energies. The imaginary strength of the emperical potential decreases as the incident energy approaches the barrier whereas the real strength remains more or less f at with a slight declining trend. The energy variations of the effective potential components obtained from CDCC calculation follow the trend observed in the extracted data for ${ }^{6} \mathrm{Li}+{ }^{28} \mathrm{Si}$. But for the ${ }^{7} \mathrm{Li}+{ }^{28} \mathrm{Si}$, the nature of the theoretical prediction is largely different from the nature exhibited by the extracted experimental data. The behaviour of the calculated potential that includes the effect of breakup coupling indicates that for ${ }^{7} \mathrm{Li}+{ }^{28} \mathrm{Si}$ system breakup is not the dominating channel for coupling at lower energies. Experimental evidence of transfer channel at low energies has been reported in Ref. [19] and coupled channel calculation with such transfer channels at lower energies for ${ }^{7} \mathrm{Li}+{ }^{28} \mathrm{Si}$ need to be performed.

In Fig. 5 the barrier distribution derived from the measured fusion excitation function of ${ }^{7} \mathrm{Li}+{ }^{28} \mathrm{Si}$ is presented. The extracted data have been compared with the 1D-BPM calculation and with calculation including the rotational coupling to the frst excited state of ${ }^{28} \mathrm{Si}$. The coupling to the target excitation improves the description of the data but the effect is not so signif cant. The experimental barrier distribution, however, seem to be broad and asymmetric at the sub-barrier region. Due to a small number of data points around the barrier energy with large energy steps ( 2 $\mathrm{MeV}$ steps), the results shown are not very satisfactory to make any def nite comment on the nature of the barrier distribution. Determination of the barrier distribution following the continuum discretized coupled channel calculation is in progress.

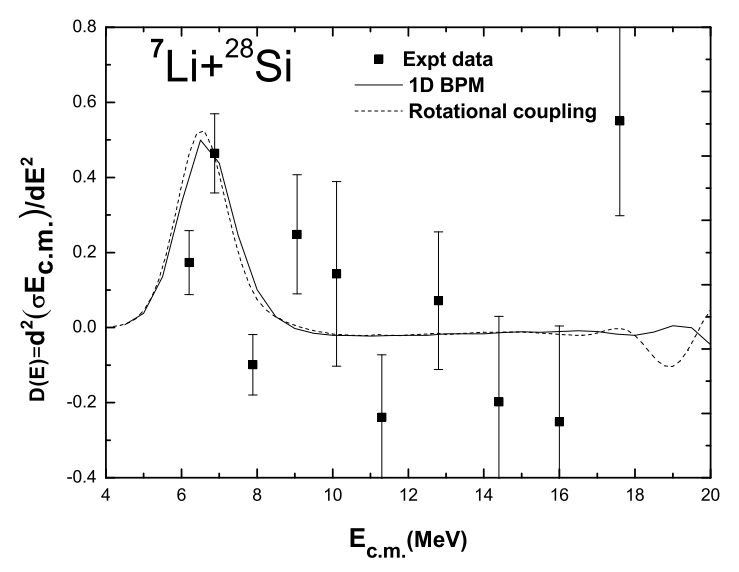

Fig. 5. Barrier distribution derived from measured fusion cross sections (solid square) compred with from ID BPM (solid line) and rotational coupling (dashed line) for ${ }^{7} \mathrm{Li}+{ }^{28} \mathrm{Si}$.

\section{References}

1. Mandira Sinha et al., Phys. Rev. C 76, (2007)027603.

2. Mandira Sinha et al., Eur. Phys.J. A 44,(2010) 403.

3. Mandira Sinha et al., Phys. Rev.C 78, (2008) 027601.

4. A. Pakou et al., Phys. Rev. C 69,(2004) 054602.

5. K. Zerva et al., Phys. Rev. C 82,(2010) 044607.

6. A. Gómez Camacho et al., Phys. Rev. C 82,(2010) 067601.

7. A. Pakou et al., Phys. Lett. B 556,(2003) 21.

8. I.J. Thompson, Comput. Phys. Rep. 7, (1988) 167.

9. F.A. Souza, et al., Phys. Rev. C 75,(2007) 044601.

10. M. Biswas, et al., Nucl. Phys. A 802,(2008) 67.

11. N. Keeley, et al., Nucl. Phys. A 571,(1994) 326.

12. S. Santra, et al., Phys. Rev. C 83,(2011) 034616.

13. V. Avreigeanu et al., Phys. Rev. C 49,(1994) 2136.

14. Haxia An and Choughai Cai, Phys. C 73,(2006) 054605.

15. Xiaohua Li, et al., Nucl Phys. A789,(2007) 103.

16. G. R. Kelly et al., Phys. Rev. C 63,(2000) 024601.

17. C.Beck et al., Phys.Rev. 75,(2007) 054605.

18. K. Rusek et al., Phys.Rev. 50,(1994) 2010.

19. A. Pakou et al., Eur. Phys. J. A 39,(2009) 187. 